\title{
Moment equations for chemical reactions on interstellar dust grains
}

\begin{abstract}
A. Lipshtat and O. Biham
Racah Institute of Physics, The Hebrew University, Jerusalem 91904, Israel

Received 21 November 2002 / Accepted 16 December 2002

Abstract. While most chemical reactions in the interstellar medium take place in the gas phase, those occurring on the surfaces of dust grains play an essential role. Such surface reactions include the catalytic production of molecular hydrogen as well as more complex reaction networks producing ice mantles and various organic molecules. Chemical models based on rate equations including both gas phase and grain surface reactions have been used in order to simulate the formation of chemical complexity in interstellar clouds. For reactions in the gas phase and on large grains, rate equations, which are highly efficient to simulate, are an ideal tool. However, for small grains under low flux, the typical number of atoms or molecules of certain reactive species on a grain may go down to order one or less. In this case the discrete nature of the populations of reactive species as well as the fluctuations become dominant, thus the mean-field approximation on which the rate equations are based does not apply. Recently, a master equation approach that provides a good description of chemical reactions on interstellar dust grains was proposed. Here we present a related approach based on moment equations that can be obtained from the master equation. These equations describe the time evolution of the moments of the distribution of the population of the various chemical species on the grain. An advantage of this approach is the fact that the production rates of molecular species are expressed directly in terms of these moments. Here we use the moment equations to calculate the rate of molecular hydrogen formation on small grains. It is shown that the moment equation approach is efficient in this case in which only a single reactive species is involved. The set of equations for the case of two species is presented and the difficulties in implementing this approach for complex reaction networks involving multiple species are discussed.
\end{abstract}

Key words. ISM: molecules - molecular processes

\section{Introduction}

The chemistry of interstellar clouds consists of reactions taking place in the gas phase as well as on the surfaces of dust grains (Hartquist \& Williams 1995). Reactions that take place on dust grain surfaces include the formation of molecular hydrogen as well as reaction networks producing ice mantles and various organic molecules. The formation of $\mathrm{H}_{2}$ on dust grain surfaces is a process of fundamental importance due to the fact that $\mathrm{H}_{2}$ cannot form in the gas phase efficiently enough to account for its observed abundance (Gould \& Salpeter 1963; Hollenbach \& Salpeter 1970; Hollenbach \& Salpeter 1971; Hollenbach et al. 1971). Molecular hydrogen plays a crucial role in gravitational collapse and star formation by absorbing energetic photons and radiating the energy in the infrared, to which the cloud is more transparent, thus cooling it and enabling further collapse. Furthermore, $\mathrm{H}_{2}$ molecules are a necessary component for the initiation of chemical reaction networks that give rise to the chemical complexity observed in interstellar clouds. Therefore, the process of $\mathrm{H}_{2}$ formation has attracted the attention of both theorists (Williams 1968; Smoluchowski 1981; Smoluchowski 1983; Aronowitz \& Chang 1985; Duley \& Williams 1986; Pirronello \& Avera 1988; Sandford \& Allamandola 1993;

Send offprint requests to: $\mathrm{O}$. Biham,

e-mail: biham@phys.huji.ac.il
Takahashi et al. 1999) and experimentalists (Brackmann 1961; Schutte 1976; Pirronello et al. 1997a; Pirronello et al. 1997b; Pirronello et al. 1999; Manico et al. 2001).

The computational modeling of chemical reaction networks on dust grains in the interstellar medium is typically done using rate equation models (Pickles \& Williams 1977; d'Hendecourt 1985; Brown 1990; Brown \& Charnley 1990; Hasegawa et al. 1992; Hasegawa \& Herbst 1993a; Hasegawa \& Herbst 1993b; Caselli et al. 1993; Willacy \& Williams 1993; Shalabiea \& Greenberg 1994). These models consist of coupled ordinary differential equations that provide the time derivatives of the densities of the species involved. Integration of these equations provides the time evolution of the densities. As long as the grains are not too small and the flux is not too low, the meanfield approximation applies. In this case, rate equations are an ideal tool for the simulation of surface reactions, due to their high computational efficiency. However, in the limit of very small grains under very low flux, rate equations are not always valid. This is because they take into account only average densities and ignore the fluctuations as well as the discrete nature of the populations of the atomic and molecular species (Tielens 1995; Charnley et al. 1997; Caselli et al. 1998; Shalabiea et al. 1998; Stantcheva et al. 2001). These features become significant in the limit of small grains and low incoming flux, typically encountered in diffuse interstellar clouds where hydrogen 
recombination as well as various other grain surface reactions take place. For example, as the number of $\mathrm{H}$ atoms on a grain fluctuates in the range of 0,1 or 2 , the $\mathrm{H}_{2}$ formation rate cannot be obtained from the average number alone. This can be easily understood, since the recombination process requires at least two $\mathrm{H}$ atoms simultaneously on the surface.

Recently, a master equation approach was proposed, that is suitable for the simulation of chemical reactions on microscopic grains (Biham et al. 2001; Green et al. 2001; Biham \& Lipshtat 2002). It takes into account both the discrete nature of the $\mathrm{H}$ atoms as well as the fluctuations. In the case of hydrogen recombination, its dynamical variables are the probabilities $P_{\mathrm{H}}\left(N_{\mathrm{H}}\right)$ that there are $N_{\mathrm{H}}$ atoms on the grain at time $t$. The time derivatives $\dot{P}_{\mathrm{H}}\left(N_{\mathrm{H}}\right), N_{\mathrm{H}}=0,1,2, \ldots$ are expressed in terms of the adsorption, reaction and desorption terms. The master equation provides the time evolution of $P_{\mathrm{H}}\left(N_{\mathrm{H}}\right), N_{\mathrm{H}}=0,1,2, \ldots$, from which the recombination rate can be calculated. The master equation approach has been applied to the study of reaction networks involving multiple species (Stantcheva et al. 2002; Stantcheva \& Herbst 2002). Since the state of the system is given by the set of number densities of all species, the number of equations quickly increases with the number of reactive species. Therefore, suitable cutoffs should be imposed in order to keep the number of equations at a tractable level. A Monte Carlo approach based on the master equation was also proposed (Charnley 2001), and applied for reaction networks involving multiple species (Stantcheva \& Herbst 2002).

In this paper we present a set of moment equations that can be derived from the master equation, and use it to calculate the rate of molecular hydrogen formation on small grains. In the moment equations, the time derivatives of the moments $\left\langle N_{\mathrm{H}}^{k}\right\rangle, k=1,2, \ldots$, of the distribution $P_{\mathrm{H}}\left(N_{\mathrm{H}}\right)$ are expressed in terms of $\left\langle N_{\mathrm{H}}\right\rangle,\left\langle N_{\mathrm{H}}^{2}\right\rangle, \ldots,\left\langle N_{\mathrm{H}}^{k+1}\right\rangle$. To simulate the moment equations we impose a cutoff at a suitable value of $k$, giving rise to $k$ coupled linear differential equations. With such cutoffs, the moment equations can be used efficiently to calculate the formation rate of molecular hydrogen for both steady state and time dependent conditions. Furthermore, they provide a useful asymptotic expression for the rate of molecular hydrogen formation in the limit of very small grains. This expression can be used in order to integrate the $\mathrm{H}_{2}$ production rate over the grainsize distribution in an interstellar cloud (Mathis 1977; Draine 1984; Draine 1985; Mathis 1990; Mathis 1996; Weingartner 2001; Cox 2002), and obtain the total production rate per unit volume of the cloud.

In the case of complex reaction networks involving multiple species, the number of moment equations quickly increases as the number of reactive species grows. Furthermore, the higher moments of the distribution are large and cannot be neglected. Thus, setting up the cutoffs in order to limit the number of equations is complicated and tends to introduce significant errors. As a result, for the case of multiple species we have not been able to develop an effective computational framework based on the moment equations. Here we present the set of moment equations for two species, such as hydrogen and oxygen, where the moments take the form $\left\langle N_{\mathrm{H}}^{k} N_{\mathrm{O}}^{\ell}\right\rangle, k, l=0,1,2, \ldots$, and discuss the approximations that may be used in order to set suitable cutoffs in these equations. In spite of these difficulties, the moment equation approach has some advantages that justify further attempts to develop it for the case of multiple species. One advantage is that the moment equations are a direct generalization of the rate equations and resemble their structure. One may thus expect that they will be suitable for incorporation into models of interstellar chemistry based on rate equations. Another advantage is that in this approach, the moments, which quantify the production rate of molecules, are directly calculated unlike the case of the master equation where the calculation of moments requires further processing.

The paper is organized as follows. In Sect. 2 we consider reactions involving a single atomic specie, focusing on the case of hydrogen recombination. In Sect. 3 we consider reactions involving two atomic species, such as hydrogen and oxygen. In each of these sections we briefly describe the rate equations and master equation for the system under study and then introduce and analyze the moment equations. The results are discussed and summarized in Sect. 4.

\section{Hydrogen recombination}

Consider a diffuse interstellar cloud dominated by a density $\rho_{\mathrm{H}}$ $\left(\mathrm{cm}^{-3}\right)$ of $\mathrm{H}$ atoms, and includes some density of dust grains. The typical velocity $v_{\mathrm{H}}\left(\mathrm{cm} \mathrm{s}^{-1}\right)$ of $\mathrm{H}$ atoms in the gas phase is given by

$v_{\mathrm{H}}=\sqrt{\frac{8}{\pi} \frac{k_{B} T_{\mathrm{gas}}}{m_{\mathrm{H}}}}$,

where $m_{\mathrm{H}}=1.67 \times 10^{-24}$ (gram) is the mass of an $\mathrm{H}$ atom and $T_{\text {gas }}$ is the gas temperature (Landau \& Lifshitz 1980). To evaluate the flux of atoms onto grain surfaces we will assume, for simplicity, that the grains are spherical with a radius $r(\mathrm{~cm})$. The cross section of a grain is $\sigma=\pi r^{2}$ and its surface area is $4 \sigma$. The number of adsorption sites on a grain is denoted by $S$. The flux $F_{\mathrm{H}}$ (atoms s${ }^{-1}$ ) of $\mathrm{H}$ atoms onto the surface of a single grain is given by $F_{\mathrm{H}}=\rho_{\mathrm{H}} v_{\mathrm{H}} \sigma$. This flux can also be expressed according to $F_{\mathrm{H}}=f_{\mathrm{H}} \cdot S$, where $f_{\mathrm{H}}\left(\mathrm{ML} \mathrm{s}^{-1}\right)$ is given by $f_{\mathrm{H}}=\rho_{\mathrm{H}} v_{\mathrm{H}} /(4 s)$, and $s$ (sites $\mathrm{cm}^{-2}$ ) is the density of adsorption sites on the surface. The $\mathrm{H}$ atoms stick to the surface and hop as random walkers between adjacent sites until they either desorb as atoms or recombine into molecules. The desorption rate of an $\mathrm{H}$ atom on the surface is

$W_{\mathrm{H}}=v \cdot \exp \left(-E_{1} / k_{B} T\right)$,

where $v$ is the attempt rate (typically assumed to be $10^{12} \mathrm{~s}^{-1}$ ), $E_{1}$ is the activation energy barrier for desorption of an $\mathrm{H}$ atom and $T$ is the surface temperature. The hopping rate of an $\mathrm{H}$ atoms is

$a_{\mathrm{H}}=v \cdot \exp \left(-E_{0} / k_{B} T\right)$

where $E_{0}$ is the activation energy barrier for $\mathrm{H}$ diffusion. Here we assume that diffusion occurs only by thermal hopping, in agreement with recent experimental results (Katz et al. 1999). Throughout this paper we use the parameters obtained experimentally for amorphous carbon, namely the activation energies are $E_{0}=44.0 \mathrm{meV}$ and $E_{1}=56.7 \mathrm{meV}$ 
(Katz et al. 1999), and the density of adsorption sites on the surface is $s \simeq 5 \times 10^{13}$ (sites $\mathrm{cm}^{-2}$ ) (Biham et al. 2001). For the density of hydrogen atoms in the gas phase we take $\rho_{\mathrm{H}}=10$ (atoms $\mathrm{cm}^{-3}$ ). The gas temperature is taken as $T_{\text {gas }}=90 \mathrm{~K}$, thus $v_{\mathrm{H}}=1.37 \times 10^{5}\left(\mathrm{~cm} \mathrm{~s}^{-1}\right)$. These are typical values for diffuse interstellar clouds.

The number of $\mathrm{H}$ atoms on the grain is denoted by $N_{\mathrm{H}}$ and its expectation value under the given conditions is denoted by $\left\langle N_{\mathrm{H}}\right\rangle$. The rate $A_{\mathrm{H}}=a_{\mathrm{H}} / S$ is approximately the inverse of the time $t_{\mathrm{s}}$ required for an atom to visit nearly all the adsorption sites on the grain surface. This is due to the fact that in two dimensions the number of distinct sites visited by a random walker is linearly proportional to the number of steps, up to a logarithmic correction (Montroll \& Weiss 1965).

\subsection{Rate equation}

The rate equation describing $\mathrm{H}_{2}$ formation on dust grain surfaces takes the form

$\frac{\mathrm{d}\left\langle N_{\mathrm{H}}\right\rangle}{\mathrm{d} t}=F_{\mathrm{H}}-W_{\mathrm{H}}\left\langle N_{\mathrm{H}}\right\rangle-2 A_{\mathrm{H}}\left\langle N_{\mathrm{H}}\right\rangle^{2}$.

The first term on the right hand side describes the flux of $\mathrm{H}$ atoms, the second term describes the desorption of $\mathrm{H}$ atoms and the third term describes the diffusion and recombination. Here we assume, for simplicity, that all $\mathrm{H}_{2}$ molecules dosorb from the surface upon formation. The production rate $R_{\mathrm{H}_{2}}^{\text {grain }}$ (molecules s ${ }^{-1}$ ) of $\mathrm{H}_{2}$ molecules from a single grain is given by

$R_{\mathrm{H}_{2}}^{\text {grain }}=A_{\mathrm{H}}\left\langle N_{\mathrm{H}}\right\rangle^{2}$.

As long as $\left\langle N_{\mathrm{H}}\right\rangle \gg 1$ Eq. (5) provides a very good evaluation of the $\mathrm{H}_{2}$ formation rate. However, for very small grains and low flux, for which $\left\langle N_{\mathrm{H}}\right\rangle \simeq 1$ or less, this mean-field approach fails, and tends to overestimate the $\mathrm{H}_{2}$ production. This is because the rate equation does not take into account the discrete nature of the population of $\mathrm{H}$ atoms, and the fact that it takes at least two atoms on the surface simultaneously to enable recombination. In this limit, the master equation approach is needed in order to evaluate the $\mathrm{H}_{2}$ formation rate.

\subsection{Master equation}

We will now describe the master equation for $\mathrm{H}_{2}$ formation on small interstellar grains, exposed to a flux $F_{\mathrm{H}}$ of $\mathrm{H}$ atoms. At any given time the number of $\mathrm{H}$ atoms adsorbed on the grain may be $N_{\mathrm{H}}=0,1,2, \ldots$, and the probability that there are $N_{\mathrm{H}}$ hydrogen atoms on a grain is given by $P_{\mathrm{H}}\left(N_{\mathrm{H}}\right)$, where

$\sum_{N_{\mathrm{H}}=0}^{\infty} P_{\mathrm{H}}\left(N_{\mathrm{H}}\right)=1$.

The time derivatives of these probabilities, $\dot{P}_{\mathrm{H}}\left(N_{\mathrm{H}}\right)$, are given by

$$
\begin{aligned}
\dot{P}_{\mathrm{H}}(0)= & -F_{\mathrm{H}} P_{\mathrm{H}}(0)+W_{\mathrm{H}} P_{\mathrm{H}}(1)+2 \cdot 1 \cdot A_{\mathrm{H}} P_{\mathrm{H}}(2) \\
\dot{P}_{\mathrm{H}}(1)= & F_{\mathrm{H}}\left[P_{\mathrm{H}}(0)-P_{\mathrm{H}}(1)\right] \\
& +W_{\mathrm{H}}\left[2 P_{\mathrm{H}}(2)-P_{\mathrm{H}}(1)\right]+3 \cdot 2 \cdot A_{\mathrm{H}} P_{\mathrm{H}}(3)
\end{aligned}
$$

$$
\begin{aligned}
\dot{P}_{\mathrm{H}}\left(N_{\mathrm{H}}\right)= & F_{\mathrm{H}}\left[P_{\mathrm{H}}\left(N_{\mathrm{H}}-1\right)-P_{\mathrm{H}}\left(N_{\mathrm{H}}\right)\right] \\
& +W_{\mathrm{H}}\left[\left(N_{\mathrm{H}}+1\right) P_{\mathrm{H}}\left(N_{\mathrm{H}}+1\right)-N_{\mathrm{H}} P_{\mathrm{H}}\left(N_{\mathrm{H}}\right)\right] \\
& +A_{\mathrm{H}}\left[\left(N_{\mathrm{H}}+2\right)\left(N_{\mathrm{H}}+1\right) P_{\mathrm{H}}\left(N_{\mathrm{H}}+2\right)\right. \\
& \left.-N_{\mathrm{H}}\left(N_{\mathrm{H}}-1\right) P_{\mathrm{H}}\left(N_{\mathrm{H}}\right)\right] .
\end{aligned}
$$

Each of these equations includes three terms. The first term describes the effect of the incoming flux $F_{\mathrm{H}}$ on the probabilities. The probability $P_{\mathrm{H}}\left(N_{\mathrm{H}}\right)$ increases when an $\mathrm{H}$ atom is adsorbed on a grain that already has $N_{\mathrm{H}}-1$ adsorbed atoms (at a rate of $F_{\mathrm{H}} P_{\mathrm{H}}\left(N_{\mathrm{H}}-1\right)$ ), and decreases when a new atom is adsorbed on a grain with $N_{\mathrm{H}}$ atoms on it (at a rate of $F_{\mathrm{H}} P_{\mathrm{H}}\left(N_{\mathrm{H}}\right)$ ). The second term includes the effect of desorption. An $\mathrm{H}$ atom that is desorbed from a grain with $N_{\mathrm{H}}$ atoms, decreases the probability $P_{\mathrm{H}}\left(N_{\mathrm{H}}\right)$ (at a rate of $N_{\mathrm{H}} W_{\mathrm{H}} P_{\mathrm{H}}\left(N_{\mathrm{H}}\right)$ ), and increases the probability $P_{\mathrm{H}}\left(N_{\mathrm{H}}-1\right)$ at the same rate. The third term describes the effect of recombination on the number of adsrobed $\mathrm{H}$ atoms. The production of one molecule reduces this number from $N_{\mathrm{H}}$ to $N_{\mathrm{H}}-2$. For one pair of $\mathrm{H}$ atoms the recombination rate is proportional to the sweeping rate $A_{\mathrm{H}}$ multiplied by 2 since both atoms are mobile simultaneously. This rate is multiplied by the number of possible pairs of atoms, namely $N_{\mathrm{H}}\left(N_{\mathrm{H}}-1\right) / 2$. Note that the equations for $\dot{P}_{\mathrm{H}}(0)$ and $\dot{P}_{\mathrm{H}}(1)$ do not include all the terms, because at least one $\mathrm{H}$ atom is required for desorption to occur and at least two for recombination. The rate of formation of $\mathrm{H}_{2}$ molecules, $R_{\mathrm{H}_{2}}^{\text {grain }}$ (molecules s ${ }^{-1}$ ), on the surface of a single grain is thus given by

$R_{\mathrm{H}_{2}}^{\text {grain }}=A_{\mathrm{H}}\left(\left\langle N_{\mathrm{H}}^{2}\right\rangle-\left\langle N_{\mathrm{H}}\right\rangle\right)$

where

$\left\langle N_{\mathrm{H}}^{k}\right\rangle=\sum_{N_{\mathrm{H}}=0}^{\infty} N_{\mathrm{H}}^{k} P_{\mathrm{H}}\left(N_{\mathrm{H}}\right)$

is the $k$ th moment of the distribution.

The time dependence of $R_{\mathrm{H}_{2}}^{\text {grain }}$ can be obtained by numerically integrating Eqs. (7) using a standard Runge-Kutta stepper. For the case of steady state, namely $\dot{P}_{\mathrm{H}}\left(N_{\mathrm{H}}\right)=0$ for all $N_{\mathrm{H}}$, an analytical solution for $P_{\mathrm{H}}\left(N_{\mathrm{H}}\right)$ is available, given in terms of $A_{\mathrm{H}} / W_{\mathrm{H}}$ and $W_{\mathrm{H}} / F_{\mathrm{H}}$ (Green et al. 2001; Biham \& Lipshtat 2002).

\subsection{Moment equations}

By expanding the time derivatives $\left\langle\dot{N}_{\mathrm{H}}^{k}\right\rangle, k=1,2, \ldots$, using Eq. (9) and inserting the expression for $\dot{P}_{\mathrm{H}}\left(N_{\mathrm{H}}\right)$ from the master Eq. (7), one obtains the moment equations:

$$
\begin{aligned}
\frac{\mathrm{d}\left\langle N_{\mathrm{H}}\right\rangle}{\mathrm{d} t}= & F_{\mathrm{H}}+\left(-W_{\mathrm{H}}+2 A_{\mathrm{H}}\right)\left\langle N_{\mathrm{H}}\right\rangle-2 A_{\mathrm{H}}\left\langle N_{\mathrm{H}}^{2}\right\rangle \\
\frac{\mathrm{d}\left\langle N_{\mathrm{H}}^{2}\right\rangle}{\mathrm{d} t}= & F_{\mathrm{H}}+\left(2 F_{\mathrm{H}}+W_{\mathrm{H}}-4 A_{\mathrm{H}}\right)\left\langle N_{\mathrm{H}}\right\rangle \\
& +\left(8 A_{\mathrm{H}}-2 W_{\mathrm{H}}\right)\left\langle N_{\mathrm{H}}^{2}\right\rangle-4 A_{\mathrm{H}}\left\langle N_{\mathrm{H}}^{3}\right\rangle
\end{aligned}
$$




$$
\begin{aligned}
\frac{\mathrm{d}\left\langle N_{\mathrm{H}}^{3}\right\rangle}{\mathrm{d} t}= & F_{\mathrm{H}}+\left(3 F_{\mathrm{H}}-W_{\mathrm{H}}+8 A_{\mathrm{H}}\right)\left\langle N_{\mathrm{H}}\right\rangle \\
& +\left(3 F_{\mathrm{H}}+3 W_{\mathrm{H}}-20 A_{\mathrm{H}}\right)\left\langle N_{\mathrm{H}}^{2}\right\rangle \\
& +\left(18 A_{\mathrm{H}}-3 W_{\mathrm{H}}\right)\left\langle N_{\mathrm{H}}^{3}\right\rangle-6 A_{\mathrm{H}}\left\langle N_{\mathrm{H}}^{4}\right\rangle \\
& \vdots \\
\frac{\mathrm{d}\left\langle N_{\mathrm{H}}^{k}\right\rangle}{\mathrm{d} t}= & F_{\mathrm{H}}\left\langle\left(1+N_{\mathrm{H}}\right)^{k}-N_{\mathrm{H}}^{k}\right\rangle \\
& +W_{\mathrm{H}}\left\langle N_{\mathrm{H}}\left[\left(N_{\mathrm{H}}-1\right)^{k}-N_{\mathrm{H}}^{k}\right]\right\rangle \\
& +A_{\mathrm{H}}\left\langle N_{\mathrm{H}}\left(N_{\mathrm{H}}-1\right)\left[\left(N_{\mathrm{H}}-2\right)^{k}-N_{\mathrm{H}}^{k}\right]\right\rangle .
\end{aligned}
$$

This is a set of coupled differential equations, that are linear in the moments $\left\langle N_{\mathrm{H}}^{k}\right\rangle$. The equation for the $k$ th moment depends on all the moments up the the $(k+1)$ th order. A numerical integration of these equations would provide the values of these moments at any given time. The $\mathrm{H}_{2}$ production rate, that depends only on the first and second moments, could then be obtained. However, the difficulty is that we need to truncate this set of equations, say after the equation of the $k$ th moment. The $(k+1)$ th moment appears in this equation and we end up with more unknowns than equations. The $(k+1)$ th moment should then be approximated as a function of the first $k$ moments. The approximation should be examined carefully since $\left\langle N_{\mathrm{H}}^{k+1}\right\rangle$ is not a small quantity (unlike the case when the master equation is truncated). In fact, due to the discreteness of $N_{\mathrm{H}}$, the moments increase monotonically with their order, namely $\left\langle N_{\mathrm{H}}^{k}\right\rangle \leq\left\langle N_{\mathrm{H}}^{k+1}\right\rangle, k=1,2, \ldots$ (and the $\leq$ symbol is replaced by the $<$ symbol if $P_{\mathrm{H}}\left(N_{\mathrm{H}}\right)>0$ for at least one value of $\left.N_{\mathrm{H}}>1\right)$.

\subsubsection{Setting the cutoffs}

Consider the case in which only up to $k$ hydrogen atoms are allowed to reside simultaneously on the surface of a grain. The moments of the resulting truncated distribution $P_{\mathrm{H}}\left(N_{\mathrm{H}}\right)$ will be

$$
\begin{aligned}
\left\langle N_{\mathrm{H}}^{1}\right\rangle= & P_{\mathrm{H}}(1)+2 P_{\mathrm{H}}(2)+\ldots+k P_{\mathrm{H}}(k) \\
\left\langle N_{\mathrm{H}}^{2}\right\rangle= & P_{\mathrm{H}}(1)+4 P_{\mathrm{H}}(2)+\ldots+k^{2} P_{\mathrm{H}}(k) \\
& \vdots \\
\left\langle N_{\mathrm{H}}^{k}\right\rangle= & P_{\mathrm{H}}(1)+2^{k} P_{\mathrm{H}}(2)+\ldots+k^{k} P_{\mathrm{H}}(k) \\
\left\langle N_{\mathrm{H}}^{k+1}\right\rangle= & P_{\mathrm{H}}(1)+2^{k+1} P_{\mathrm{H}}(2)+\ldots+k^{k+1} P_{\mathrm{H}}(k) .
\end{aligned}
$$

The first $k$ equations in Eqs. (11) can be used in order to express $P_{\mathrm{H}}(1), \ldots, P_{\mathrm{H}}(k)$ in terms of $\left\langle N_{\mathrm{H}}^{1}\right\rangle, \ldots,\left\langle N_{\mathrm{H}}^{k}\right\rangle$. The results can then be plugged into the $(k+1)$ th equation in Eqs. (10), thus expressing $\left\langle N_{\mathrm{H}}^{k+1}\right\rangle$ in terms of the first $k$ moments. For example, in the case of $k=2$ we obtain the third moment as a function of the first two:

$$
\left\langle N_{\mathrm{H}}^{3}\right\rangle=3\left\langle N_{\mathrm{H}}^{2}\right\rangle-2\left\langle N_{\mathrm{H}}^{1}\right\rangle \cdot
$$

Similarly, when $P_{\mathrm{H}}\left(N_{\mathrm{H}}\right)$ is truncated after $k=3$ or $k=4$ we obtain

$$
\left\langle N_{\mathrm{H}}^{4}\right\rangle=6\left\langle N_{\mathrm{H}}^{1}\right\rangle-11\left\langle N_{\mathrm{H}}^{2}\right\rangle+6\left\langle N_{\mathrm{H}}^{3}\right\rangle
$$

and

$$
\left\langle N_{\mathrm{H}}^{5}\right\rangle=-24\left\langle N_{\mathrm{H}}^{1}\right\rangle+50\left\langle N_{\mathrm{H}}^{2}\right\rangle-35\left\langle N_{\mathrm{H}}^{3}\right\rangle+10\left\langle N_{\mathrm{H}}^{4}\right\rangle,
$$

respectively. In general, when up to $k$ hydrogen atoms are allowed to reside on a grain the $(k+1)$ th moment, $\left\langle N_{\mathrm{H}}^{k+1}\right\rangle$, can be expressed as a linear combination of the first $k$ moments according to

$\left\langle N_{\mathrm{H}}^{k+1}\right\rangle=\sum_{n=1}^{k} C_{k+1}(n)\left\langle N_{\mathrm{H}}^{n}\right\rangle$.

The coefficients $C_{k+1}(n)$ are obtained from the solution of a set of linear algebraic equations, that can be expressed in a matrix form as

$V \boldsymbol{C}=\boldsymbol{v}$.

The matrix $V$ is the Vandermonde matrix of size $k \times k$, namely, $V_{m n}=m^{n}$, where $m, n=1,2, \ldots, k$, and the vector $\boldsymbol{v}$ consists of $v_{m}=m^{k+1}, m=1,2, \ldots, k$. The desired coefficients are the elements of the vector $\boldsymbol{C}=\left[C_{k+1}(1), \ldots, C_{k+1}(k)\right]$. The coefficient $C_{k+1}(n)$ turn out to be equal to the coefficient of $x^{n}$ in the polynomial (Bender et al. 2002)

$Q_{k}(x)=-\prod_{j=0}^{k}(x-j)$.

Having an expression for $\left\langle N_{\mathrm{H}}^{k+1}\right\rangle$ as a linear combination of the first $k$ moments, we can now insert it into the last equation in Eq. (10). We then obtain a set of $k$ coupled linear differential equations for the first $k$ moments. This set of equations can be simulated using a standard Runge-Kutta or any other stepper routine. A convenient choice of initial condition may be the case of an empty grain. In this case the moment equations are initiated with $\left\langle N_{\mathrm{H}}^{n}\right\rangle=0$ for all $n \geq 1$ (while the master equation is initiated with $P_{\mathrm{H}}(0)=1$ and $P_{\mathrm{H}}(n)=0$ for $\left.n \geq 1\right)$.

\subsubsection{Calculations and results}

To determine the desirable value of the cutoff $k$, for steady state calculations using the moment equations, it is useful to first calculate $\left\langle N_{\mathrm{H}}\right\rangle$ using the rate equations (Eq. (4)) for the same parameters used in the moment equations. We found that a suitable choice for the truncation is $k=\left\lceil\left\langle N_{\mathrm{H}}\right\rangle+C\right\rceil$ where $\lceil x\rceil$ is the smallest integer which is larger than $x$. The parameter $C$ is determined such that a sufficient number of equations are included to provide a good agreement with the master equation. In all the calculations presented here we used $C=1.2$.

We have used the moment equations to simulate the hydrogen recombination process on a grain under steady state conditions, and compared the results with those of the rate equations and the master equation. The surface parameters used are of amorphous carbon. The gas density and temperature are those specified above, and the surface temperature is $T=18 \mathrm{~K}$.

The number of $\mathrm{H}$ atoms on the grain is shown in Fig. 1a as a function of the number of adsorption sites, $S$, using the rate equations (dashed line), master equation ( $O$ ) and moment equations (solid line). The production rate of $\mathrm{H}_{2}$ molecules on the surface of a single grain is shown in Fig. $1 \mathrm{~b}$ as a function of $S$. The results of the rate equations (dashed line) are simply linearly proportional to $S$. For large enough grains the 

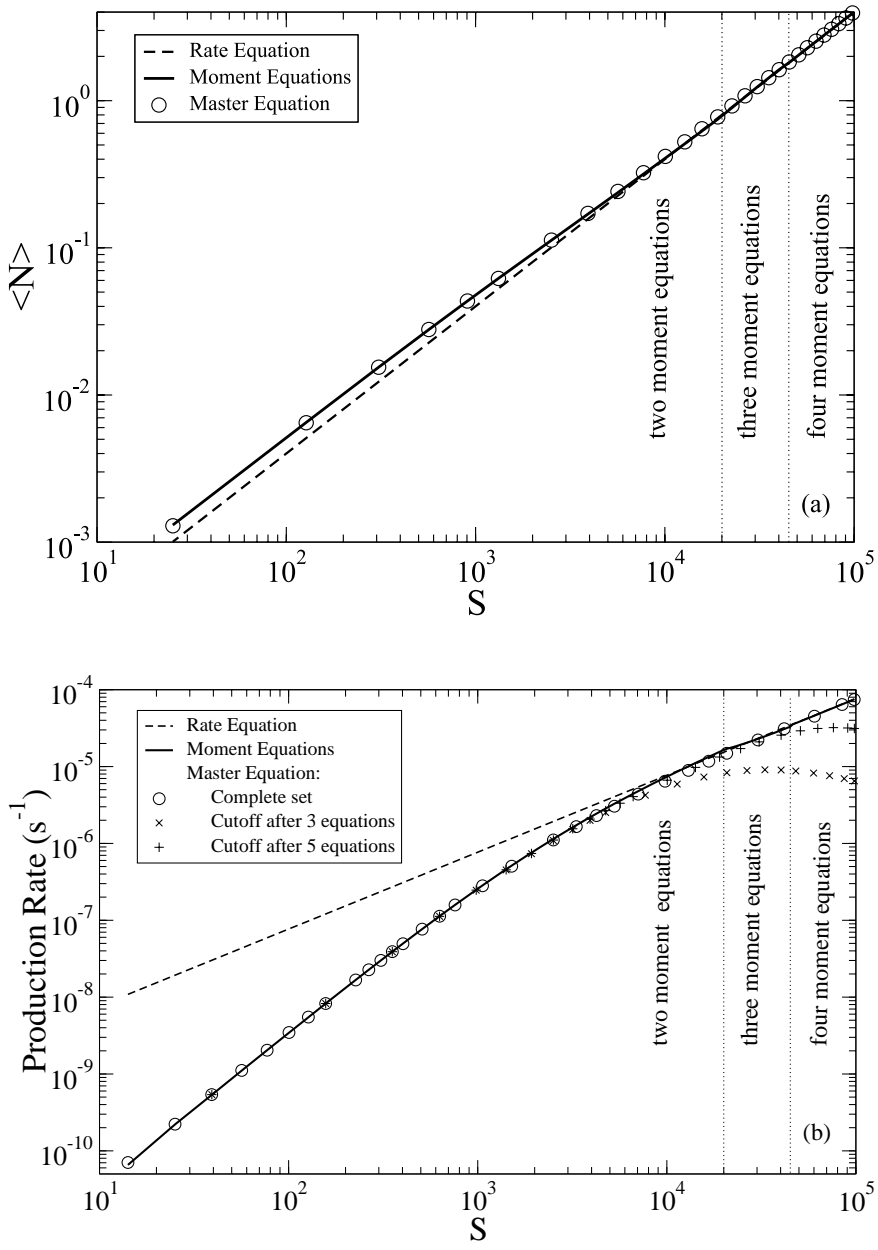

Fig. 1. a) The expectation value of the number of $H$ atoms on the grain as a function of the number of adsorption sites, $S$, on the grain, obtained from the moment equations, the master equation and the rate equations; b) The production rate $R_{\mathrm{H}_{2}}^{\text {grain }}$ of molecular hydrogen on a single grain of $S$ surface adsorption sites. The results of the moment equations (solid line) are in perfect agreement with those of the master equation (O), while the rate equations (dashed line) over-estimate the production rate for small grains. The number of moment equations used for each range of grain sizes is specified.

results of the rate equations coincide with those of the master equation $(\circ)$ and the moments equations (solid line). For small grains, the results of the moment equations are in perfect agreement with the master equation, while the rate equations greatly over-estimate the production rate. In the simulations of the moment equations, the cutoff was determined according the the procedure specified above. The number of equations used in each range of grain sizes is specified. Note that using this procedure we obtain a perfect agreement between the moment equations and the master equation for the entire range of grain sizes. Results are also shown for the master equation with cutoffs after the third equation $(\times)$ and after the fifth equation $(+)$. It is shown that while the sets of two, three and four moment equations provide a perfect agreement with the complete master equation in the specified domains, the master equation with cutoffs (including at least as many equations) exhibits significant deviations.
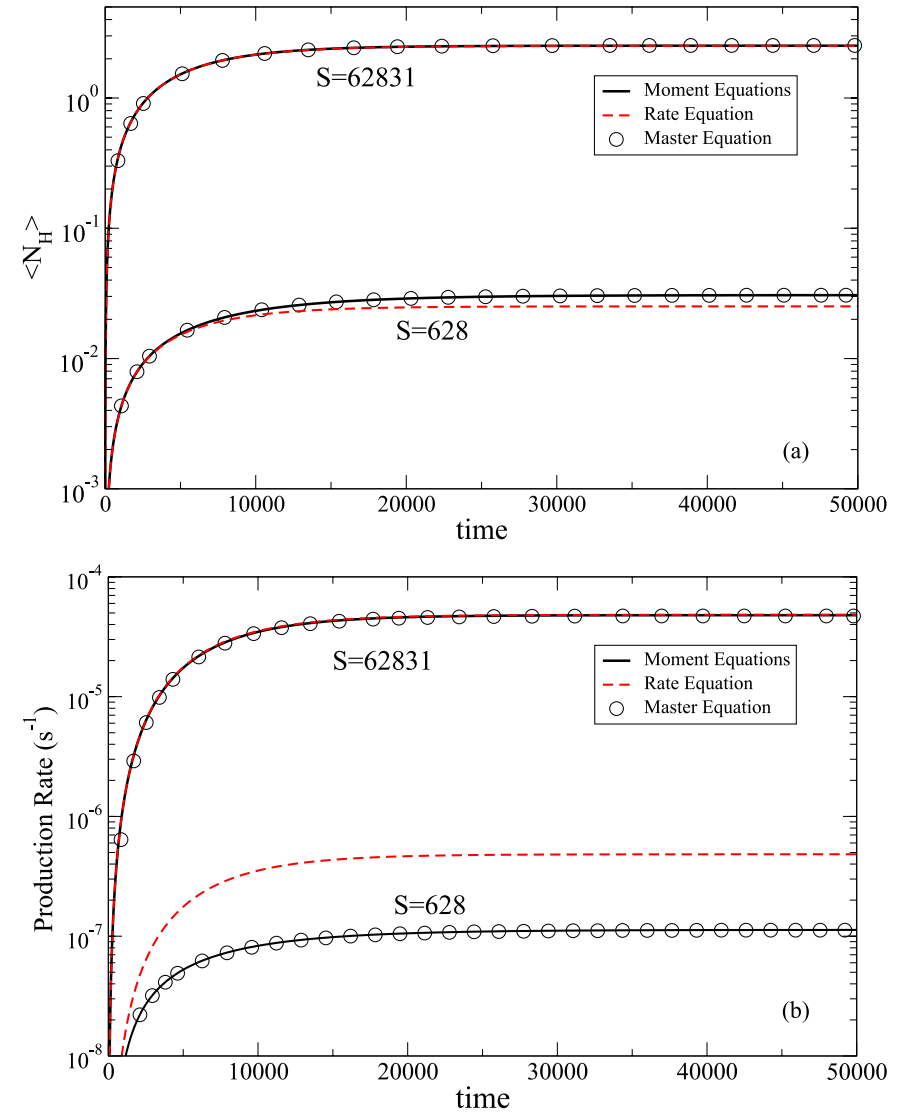

Fig. 2. a) The expectation value for the number of $\mathrm{H}$ atoms on the grain vs. time; and b) the production rate $R_{\mathrm{H}_{2}}^{\text {grain }}$ of molecular hydrogen vs. time, for a large grain ( $S=62,831$ sites) and for a small grain ( $S=628$ sites). The initial condition is of an empty grain surface, while the surface parameters, the temperature and the flux are identical to those used in Fig. 1. For the large grain, it is found that the moment equations (solid line), master equation $(\mathrm{O})$ and the rate equations (dashed line) are all in perfect agreement even during the transient time. For the small grain, the set of two moment equations is in perfect agreement with the complete master equation, while the rate equations deviate significantly.

In the limit of very small grains, only two moment equations are needed in order to obtain perfect agreement with the master equation. These equations are derived from the first two moment equations in Eqs. (10) and the cutoff condition given in Eq. (12). They take the form

$$
\begin{aligned}
& \frac{\mathrm{d}\left\langle N_{\mathrm{H}}\right\rangle}{\mathrm{d} t}=F_{\mathrm{H}}+\left(2 A_{\mathrm{H}}-W_{\mathrm{H}}\right)\left\langle N_{\mathrm{H}}\right\rangle-2 A_{\mathrm{H}}\left\langle N_{\mathrm{H}}^{2}\right\rangle \\
& \frac{\mathrm{d}\left\langle N_{\mathrm{H}}^{2}\right\rangle}{\mathrm{d} t}=F_{\mathrm{H}}+\left(2 F_{\mathrm{H}}+W_{\mathrm{H}}+4 A_{\mathrm{H}}\right)\left\langle N_{\mathrm{H}}\right\rangle-\left(4 A_{\mathrm{H}}+2 W_{\mathrm{H}}\right)\left\langle N_{\mathrm{H}}^{2}\right\rangle .
\end{aligned}
$$

We find that these equations are dynamically stable and can be used for time-dependent simulations. In Fig. 2 we present the results of time-dependent simulations using Eq. (18) for a large grain $(S=62,831$ sites $)$ and for a small grain $(S=$ 628 sites). The expectation value $\left\langle N_{\mathrm{H}}\right\rangle$ vs. time is shown in Fig. $2 \mathrm{a}$, and the production rate $R_{\mathrm{H}_{2}}^{\text {grain }}$ of molecular hydrogen is shown in Fig. 2b. The initial condition is of an empty grain surface, while the surface parameters, the temperature and the 


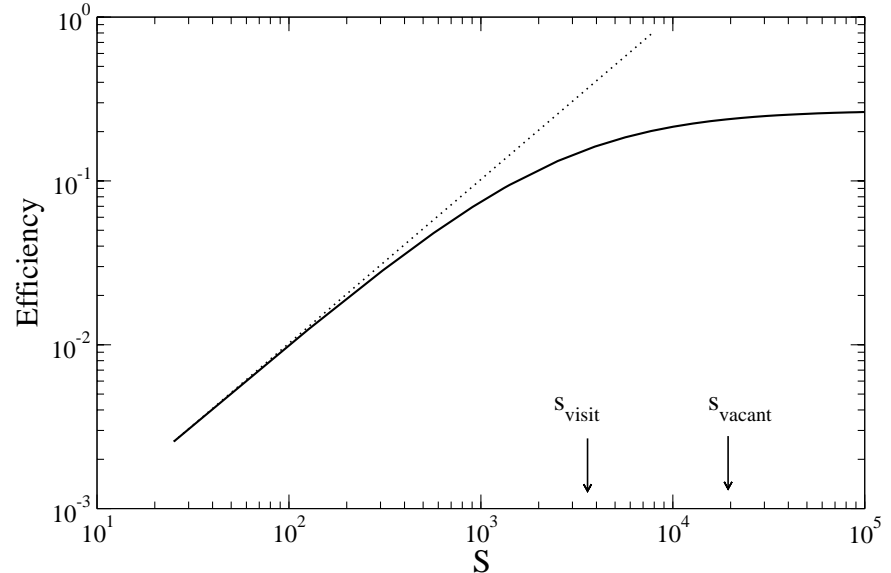

Fig. 3. The efficiency $\eta(S)$ of molecular hydrogen formation on a grain of $S$ assorption sites is presented. It is shown that in the limit of small grains the efficiency depends linearly on $S$, while for large grains it saturates towards the efficiency predicted by the rate equations and becomes independent of $S$.

flux are identical to those used in Fig. 1. For the large grain, it is found that the moment equations, master equation and the rate equation are all in perfect agreement even during the transient time. For the small grain, the set of two moment equations is in perfect agreement with the complete master equation, while the rate equation deviates significantly. For steady state conditions, Eqs. (18) can be solved exactly, giving rise to

$$
\begin{aligned}
\left\langle N_{\mathrm{H}}\right\rangle & =\frac{F_{\mathrm{H}}\left(A_{\mathrm{H}}+W_{\mathrm{H}}\right)}{2 A_{\mathrm{H}} F_{\mathrm{H}}+W_{\mathrm{H}} A_{\mathrm{H}}+W_{\mathrm{H}}^{2}} \\
\left\langle N_{\mathrm{H}}^{2}\right\rangle & =\frac{F_{\mathrm{H}}\left(F_{\mathrm{H}}+A_{\mathrm{H}}+W_{\mathrm{H}}\right)}{2 A_{\mathrm{H}} F_{\mathrm{H}}+W_{\mathrm{H}} A_{\mathrm{H}}+W_{\mathrm{H}}^{2}} .
\end{aligned}
$$

A simple exact expression for the production rate $R_{\mathrm{H}_{2}}^{\text {grain }}$, of molecular hydrogen, given by Eq. (8), can now be obtained for steady state conditions in the limit of small grains and low flux. It takes the form

$R_{\mathrm{H}_{2}}^{\text {grain }}=\frac{f_{\mathrm{H}}^{2}}{W_{\mathrm{H}}}\left[\frac{S^{2}}{1+\frac{S}{a_{\mathrm{H}} / W_{\mathrm{H}}}+2 \frac{S}{W_{\mathrm{H}} / f_{\mathrm{H}}}}\right]$.

We observe that, as long as $S$ is larger than both $s_{\text {visit }}=a_{\mathrm{H}} / W_{\mathrm{H}}$ (the number of sites that an $\mathrm{H}$ atom typically visits before it desorbs) and $s_{\mathrm{vacant}}=W_{\mathrm{H}} / f_{\mathrm{H}}$ (the typical number of vacant sites per adsorbed $\mathrm{H}$ atom), the production rate is linearly proportional to $S$, and the grain size does not play any special role (Biham \& Lipshtat 2002). In the case of small grains for which $S$ is smaller than both $s_{\text {visit }}$ and $s_{\text {vacant }}$ the production rate is reduced and becomes proportional to $S^{2}$. The production efficiency $\eta(S)=2 R_{\mathrm{H}_{2}}^{\text {grain }} / F_{\mathrm{H}}$ then takes the form

$\eta(S)=\frac{2 f_{\mathrm{H}}}{W_{\mathrm{H}}}\left[\frac{S}{1+\frac{S}{a_{\mathrm{H}} / W_{\mathrm{H}}}+2 \frac{S}{W_{\mathrm{H}} / f_{\mathrm{H}}}}\right]$.

In Fig. 3 we present the production efficiency, given by Eq. (21), as a function of $S$ under the same conditions as those used in Fig. 1. For very small grains, namely for $S<$ $\min \left\{s_{\text {visit }}, s_{\text {vacant }}\right\}$, the efficiency $\eta(S)$ is linearly proportional

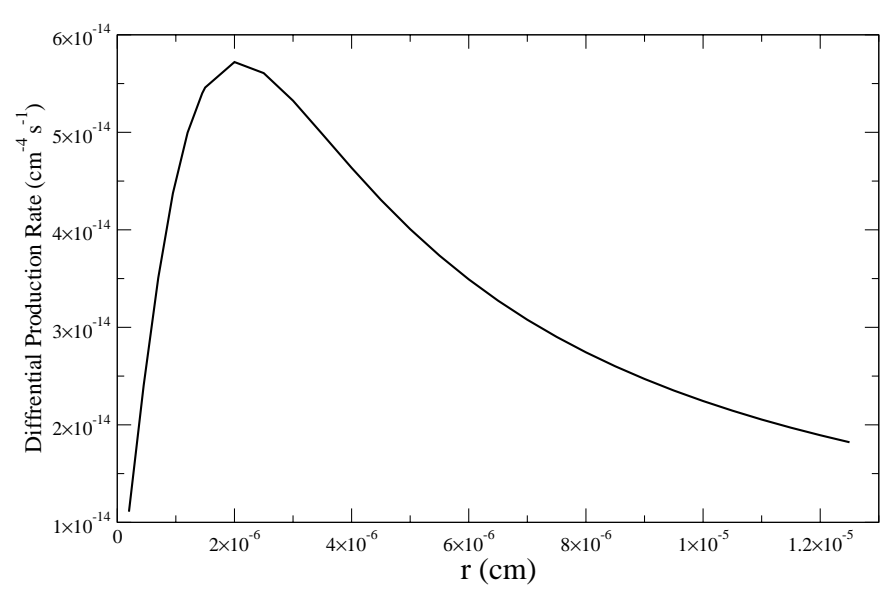

Fig. 4. The differential production rate of molecular hydrogen, given by the integrand in Eq. (22) vs. the grain radius, $r$. The largest contribution comes from very small grains, of sizes around $20 \mathrm{~nm}$. For larger grains the contribution decreases due to the fact that their total surface area is smaller. For smaller grains the contribution decreases due to the reduction in the recombination efficiency.

to $S$, while for $S>\max \left\{s_{\text {visit }}, s_{\text {vacant }}\right\}$ it saturates and coincides with the rate equation result that is independent of $S$.

These results can now be used in order to evaluate the total production of molecular hydrogen per unit volume of the cloud from grains of all sizes. Consider a cloud in which the grain size distribution is given by $\rho_{\mathrm{gr}}(r)\left(\mathrm{cm}^{-4}\right)$ in the range $r_{\text {min }}<$ $r<r_{\max }$, where $r_{\min }$ and $r_{\max }$ are the lower and upper cutoffs of the distribution (here $\rho_{\mathrm{gr}}(r) \mathrm{d} r\left(\mathrm{~cm}^{-3}\right)$ is the number density of grains of sizes in the range $(r, r+\mathrm{d} r))$. The formation rate $R_{\mathrm{H}_{2}}$ $\left(\mathrm{cm}^{-3} \mathrm{~s}^{-1}\right)$ of molecular hydrogen in the cloud is given by

$R_{\mathrm{H}_{2}}=\rho_{\mathrm{H}} v_{\mathrm{H}} \int_{r_{\text {min }}}^{r_{\max }} \pi r^{2} \rho_{\mathrm{gr}}(r) \eta(S) \mathrm{d} r$

where $\eta(S)$ is given by Eq. (21) and $S=4 \pi r^{2} s$.

To demonstrate these ideas we will consider a simple example in which the grain size distribution exhibits a power-law behavior of the form

$\rho_{\mathrm{gr}}(r)=\frac{K}{r^{\alpha}}$

where $\alpha=3$. The special property of this distribution is that the total grain mass is equally distributed in the entire range of grain sizes $r_{\min } \leq r \leq r_{\max }$. Unlike the mass, the total surface area of all grains in the size range between $r$ and $r+\mathrm{d} r$, scales like $r^{-1}$, namely most of the grain surface area is on the small grains. The prefactor $K$ is determined by the condition that the total number density of grains is smaller than $\rho_{\mathrm{H}}$ by a factor of $10^{-12}$. Under this condition $K=2 \times 10^{-11} /\left(r_{\min }^{-2}-r_{\max }^{-2}\right)$. In this case the integral can be easily evaluated and the result is

$R_{\mathrm{H}_{2}}=\frac{4 \pi^{2} K \rho_{\mathrm{H}} v_{\mathrm{H}} f_{\mathrm{H}} s}{W_{\mathrm{H}} B} \ln \left(\frac{B r_{\max }^{2}+1}{B r_{\text {min }}^{2}+1}\right)$

where $B=4 \pi s\left[W_{\mathrm{H}} / a_{\mathrm{H}}+2 f_{\mathrm{H}} / W_{\mathrm{H}}\right]$. To evaluate the contribution of grains of different sizes to the total production rate of molecular hydrogen, we show in Fig. 4 the differential production rate, given by the integrand in Eq. (22), vs. the grain radius, $r$, where $r_{\min }=2 \times 10^{-7}(\mathrm{~cm})$ and $r_{\max }=1.25 \times 10^{-5}(\mathrm{~cm})$. 
Starting from the limit of large grains, the differential production increases with decreasing grain size due to the larger total surface area of smaller grains. However, below some grain size the recombination efficiency decreases and the differential production rate starts to decrease. We observe that under these conditions the largest contribution comes from extremely small grains of sizes around $20 \mathrm{~nm}$. Observation indicate that the exponent in Eq. (23) is $\alpha=3.5$ (see e.g. Weingartner \& Draine 2001). Therefore, the contribution of very small grains to molecular hydrogen formation may be even more dominant than in the analysis shown here.

Observations indicate that the temperatures of dust grains in diffuse clouds are typically in the range between 10-20 K. The dependence of the molecular hydrogen formation process on the surface temperature of the grains is presented in Fig. 5. The average number of $\mathrm{H}$ atoms on the surface of a very small grain of radius $r=10^{-6}(\mathrm{~cm})$ is shown in Fig. 5a. The production rate of molecular hydrogen on such grain is shown in Fig. 5b. The results of the moment equations (solid line) and the master equation $(\circ)$ coincide perfectly, while the rate equations (dashed line) significantly overestimate the production rate for temperatures higher than $16 \mathrm{~K}$. As the temperature increases above $T=16 \mathrm{~K}$, desorption becomes faster and the production rate decreases. Below $16 \mathrm{~K}$ the production rate saturates and becomes independent of the temperature within the temperature window of $12-16 \mathrm{~K}$. Below $T=12 \mathrm{~K}$ the production rate decreases sharply due to the lower mobility of $\mathrm{H}$ atoms on the surface Katz et al. (1999).

\section{Reactions involving two species}

Observations in the past two decades provide evidence that chemical reaction networks on dust grain surfaces go much beyond the production of molecular hydrogen. In particular, ice mantles that consist of $\mathrm{H}_{2} \mathrm{O}$ and $\mathrm{CO}$, as well as more complex organic molecules, form on grain surfaces.

Here we consider a surface reaction network involving hydrogen and oxygen (Caselli et al. 1998). In this system surface reactions produce $\mathrm{H}_{2}, \mathrm{O}_{2}, \mathrm{OH}$ and $\mathrm{H}_{2} \mathrm{O}$ molecules. While the hydrogen molecules typically desorb upon formation or shortly later, the heavier species tend to remain on the surface. Some of them, such as $\mathrm{OH}$ may then participate in further reactions. However, in the model presented below we assume, for simplicity, that the reaction products do not participate in further chemical reactions (namely, $\mathrm{H}_{2} \mathrm{O}$ is not produced). In practice, we consider these molecules as if they all desorb from the surface upon formation.

\subsection{Rate equations}

The rate equations that describe the chemical reactions on dust grains in our simplified hydrogen-oxygen system are

$$
\begin{aligned}
& \frac{\mathrm{d}\left\langle N_{\mathrm{H}}\right\rangle}{\mathrm{d} t}=F_{\mathrm{H}}-W_{\mathrm{H}}\left\langle N_{\mathrm{H}}\right\rangle-2 A_{\mathrm{H}}\left\langle N_{\mathrm{H}}\right\rangle^{2}-\left(A_{\mathrm{H}}+A_{\mathrm{O}}\right)\left\langle N_{\mathrm{H}}\right\rangle\left\langle N_{\mathrm{O}}\right\rangle \\
& \frac{\mathrm{d}\left\langle N_{\mathrm{O}}\right\rangle}{\mathrm{d} t}=F_{\mathrm{O}}-W_{\mathrm{O}}\left\langle N_{\mathrm{O}}\right\rangle-2 A_{\mathrm{O}}\left\langle N_{\mathrm{O}}\right\rangle^{2}-\left(A_{\mathrm{H}}+A_{\mathrm{O}}\right)\left\langle N_{\mathrm{H}}\right\rangle\left\langle N_{\mathrm{O}}\right\rangle
\end{aligned}
$$
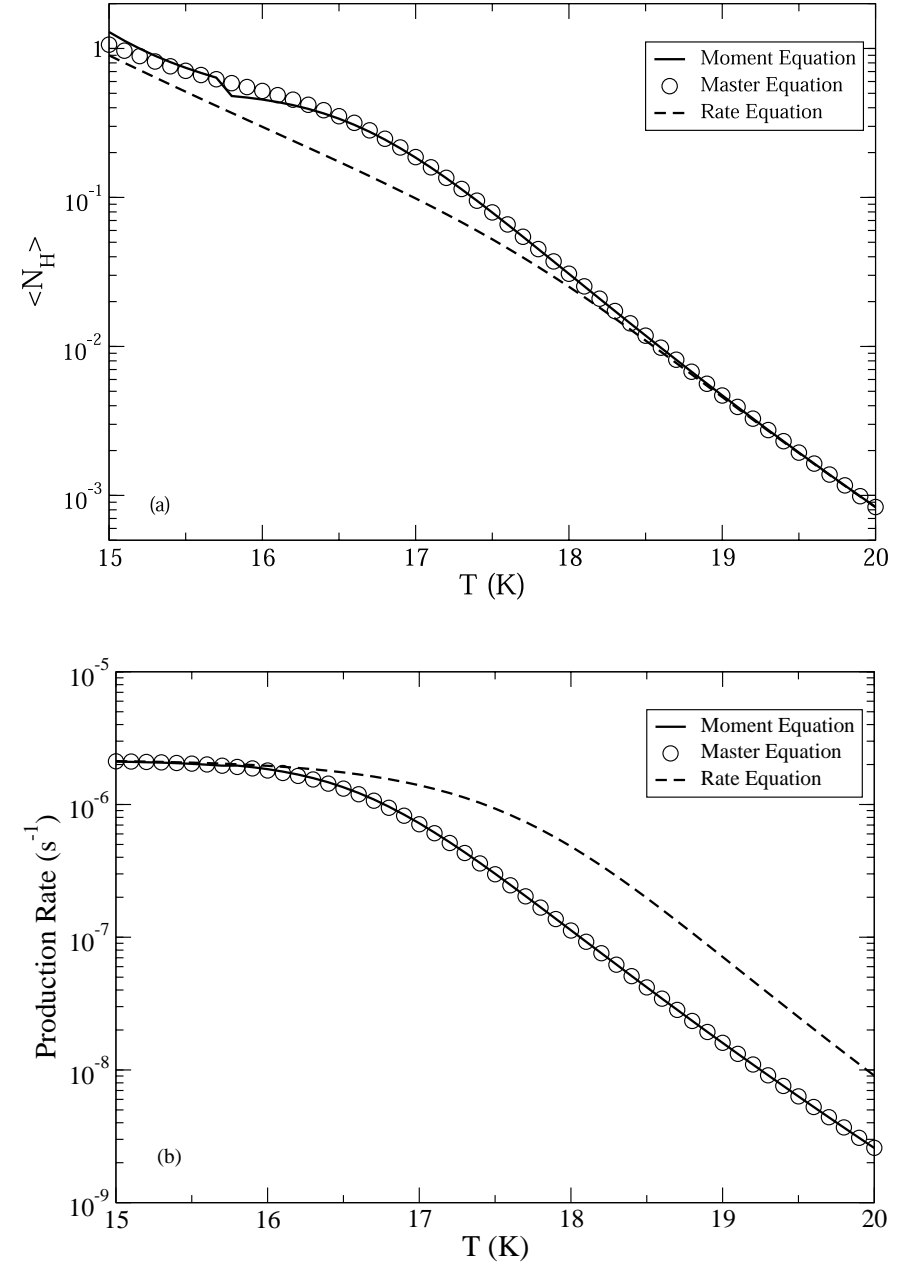

Fig. 5. The average number of $H$ atoms on the grain surface a), and the production rate b) for a grain of radius $r=10^{-6}(\mathrm{~cm})$ as a function of the grain temperature. The results of the moment equations (solid line) and the master equation (O) coincide perfectly, while the rate equations (dashed line) significantly overestimate the production rate for temperatures higher than $16 \mathrm{~K}$. As the temperature increases above $T=16 \mathrm{~K}$, desorption becomes faster and the production rate decreases. Below $16 \mathrm{~K}$ the production rate saturates and becomes independent of the temperature within the temperature window of $12-16 \mathrm{~K}$.

where $N_{\mathrm{O}}$ is the number of oxygen atoms on the grain and $A_{\mathrm{O}}=a_{\mathrm{O}} / S$ is their sweeping rate. Here we denote the density of the $\mathrm{O}$ atoms in the gas phase by $\rho_{\mathrm{O}}$. Their velocity, $v_{\mathrm{O}}$, is determined by Eq. (1) (replacing $m_{\mathrm{H}}$ by the mass $m_{\mathrm{O}}$ of an oxygen atom) with the same temperature as that of the hydrogen gas. The flux of $\mathrm{O}$ atoms adsorbed on the grain surface is given by $F_{\mathrm{O}}=\rho_{\mathrm{O}} v_{\mathrm{O}} \sigma$ (atoms s${ }^{-1}$ ). The desorption rate $W_{\mathrm{O}}$ is given by an expression analogous to Eq. (2) with the same $v$ and with $E_{1}$ replaced by the activation energy for desorption of $\mathrm{O}$ atoms. The hopping rate $a_{\mathrm{O}}$ of the $\mathrm{O}$ atoms is given by an expression analogous to Eq. (3), with $E_{0}$ replaced by the activation energy for hopping of $\mathrm{O}$ atoms.

As long as the grains are not too small and the fluxes are not too low, such that $N_{\mathrm{H}} \gg 1$ and $N_{\mathrm{O}} \gg 1$ these rate equations evaluate correctly the formation rates of all the reaction products. However, when the typical numbers of $\mathrm{H}$ or $\mathrm{O}$ atoms on the grain go down to order one, these equations become 
unsuitable and exhibit significant deviations from the actual production rates.

\subsection{Master equation}

The master equation that describes the hydrogen-oxygen system consists of a two dimensional matrix of equations for the time derivatives of $P_{\mathrm{H} \& \mathrm{O}}\left(N_{\mathrm{H}}, N_{\mathrm{O}}\right)$. It takes the form

$$
\begin{aligned}
\dot{P}_{\mathrm{H} \& \mathrm{O}}\left(N_{\mathrm{H}}, N_{\mathrm{O}}\right)= & F_{\mathrm{H}}\left[P_{\mathrm{H} \& \mathrm{O}}\left(N_{\mathrm{H}}-1, N_{\mathrm{O}}\right)\right. \\
& \left.-P_{\mathrm{H} \& \mathrm{O}}\left(N_{\mathrm{H}}, N_{\mathrm{O}}\right)\right] \\
& +F_{\mathrm{O}}\left[P_{\mathrm{H} \& \mathrm{O}}\left(N_{\mathrm{H}}, N_{\mathrm{O}}-1\right)\right. \\
& \left.-P_{\mathrm{H} \& \mathrm{O}}\left(N_{\mathrm{H}}, N_{\mathrm{O}}\right)\right] \\
& +W_{\mathrm{H}}\left[\left(N_{\mathrm{H}}+1\right) P_{\mathrm{H} \& \mathrm{O}}\left(N_{\mathrm{H}}+1, N_{\mathrm{O}}\right)\right. \\
& \left.-N_{\mathrm{H}} P_{\mathrm{H} \& \mathrm{O}}\left(N_{\mathrm{H}}, N_{\mathrm{O}}\right)\right] \\
& +W_{\mathrm{O}}\left[\left(N_{\mathrm{O}}+1\right) P_{\mathrm{H} \& \mathrm{O}}\left(N_{\mathrm{H}}, N_{\mathrm{O}}+1\right)\right. \\
& \left.-N_{\mathrm{O}} P_{\mathrm{H} \& \mathrm{O}}\left(N_{\mathrm{H}}, N_{\mathrm{O}}\right)\right] \\
& +A_{\mathrm{H}}\left[\left(N_{\mathrm{H}}+2\right)\left(N_{\mathrm{H}}+1\right) P_{\mathrm{H} \& \mathrm{O}}\left(N_{\mathrm{H}}+2, N_{\mathrm{O}}\right)\right. \\
& \left.-N_{\mathrm{H}}\left(N_{\mathrm{H}}-1\right) P_{\mathrm{H} \& \mathrm{O}}\left(N_{\mathrm{H}}, N_{\mathrm{O}}\right)\right] \\
& +A_{\mathrm{O}}\left[\left(N_{\mathrm{O}}+2\right)\left(N_{\mathrm{O}}+1\right) P_{\mathrm{H} \& \mathrm{O}}\left(N_{\mathrm{H}}, N_{\mathrm{O}}+2\right)\right. \\
& \left.-N_{\mathrm{O}}\left(N_{\mathrm{O}}-1\right) P_{\mathrm{H} \& \mathrm{O}}\left(N_{\mathrm{H}}, N_{\mathrm{O}}\right)\right] \\
& +\left(A_{\mathrm{H}}+A_{\mathrm{O}}\right) \\
& \times\left[\left(N_{\mathrm{H}}+1\right)\left(N_{\mathrm{O}}+1\right) P_{\mathrm{H} \& \mathrm{O}}\left(N_{\mathrm{H}}+1, N_{\mathrm{O}}+1\right)\right. \\
& \left.-N_{\mathrm{H}} N_{\mathrm{O}} P_{\mathrm{H} \& \mathrm{O}}\left(N_{\mathrm{H}}, N_{\mathrm{O}}\right)\right]
\end{aligned}
$$

where $N_{\mathrm{H}}, N_{\mathrm{O}}=0,1,2, \ldots$. In the equations in which $N_{\mathrm{H}}=$ 0,1 or $N_{\mathrm{O}}=0,1$, some of the terms vanish. These terms can be easily identified since their initial or final states include a negative number of $\mathrm{H}$ or $\mathrm{O}$ atoms on the grain.

\subsection{Moment equations}

The moment equations for the hydrogen-oxygen system take the form

$$
\begin{aligned}
\frac{\mathrm{d}\left\langle N_{\mathrm{H}}\right\rangle}{\mathrm{d} t}= & F_{\mathrm{H}}+\left(2 A_{\mathrm{H}}-W_{\mathrm{H}}\right)\left\langle N_{\mathrm{H}}\right\rangle-2 A_{\mathrm{H}}\left\langle N_{\mathrm{H}}^{2}\right\rangle \\
\frac{\mathrm{d}\left\langle N_{\mathrm{O}}\right\rangle}{\mathrm{d} t}= & -\left(A_{\mathrm{H}}+A_{\mathrm{O}}\right)\left\langle N_{\mathrm{H}} N_{\mathrm{O}}\right\rangle \\
& -\left(2 A_{\mathrm{O}}-W_{\mathrm{O}}\right)\left\langle N_{\mathrm{O}}\right\rangle-2 A_{\mathrm{O}}\left\langle N_{\mathrm{O}}^{2}\right\rangle \\
\frac{\mathrm{d}\left\langle N_{\mathrm{H}}^{2}\right\rangle}{\mathrm{d} t}= & F_{\mathrm{H}}+\left(2 F_{\mathrm{H}}+W_{\mathrm{H}}-4 A_{\mathrm{H}}\right)\left\langle N_{\mathrm{H}}\right\rangle \\
& +\left(-2 W_{\mathrm{H}}+8 A_{\mathrm{H}}\right)\left\langle N_{\mathrm{H}}^{2}\right\rangle \\
& -4 A_{\mathrm{H}}\left\langle N_{\mathrm{H}}^{3}\right\rangle+\left(A_{\mathrm{H}}+A_{\mathrm{O}}\right)\left\langle N_{\mathrm{H}} N_{\mathrm{O}}\right\rangle \\
& -2\left(A_{\mathrm{H}}+A_{\mathrm{O}}\right)\left\langle N_{\mathrm{H}}^{2} N_{\mathrm{O}}\right\rangle \\
\frac{\mathrm{d}\left\langle N_{\mathrm{O}}^{2}\right\rangle}{\mathrm{d} t}= & F_{\mathrm{O}}+\left(2 F_{\mathrm{O}}+W_{\mathrm{O}}-4 A_{\mathrm{O}}\right)\left\langle N_{\mathrm{O}}\right\rangle \\
+ & \left(-2 W_{\mathrm{O}}+8 A_{\mathrm{O}}\right)\left\langle N_{\mathrm{O}}^{2}\right\rangle \\
- & 4 A_{\mathrm{O}}\left\langle N_{\mathrm{O}}^{3}\right\rangle+\left(A_{\mathrm{H}}+A_{\mathrm{O}}\right)\left\langle N_{\mathrm{H}} N_{\mathrm{O}}\right\rangle \\
- & 2\left(A_{\mathrm{H}}+A_{\mathrm{O}}\right)\left\langle N_{\mathrm{H}} N_{\mathrm{O}}^{2}\right\rangle \\
= & F_{\mathrm{H}}\left\langle N_{\mathrm{O}}\right\rangle+F_{\mathrm{O}}\left\langle N_{\mathrm{H}}\right\rangle \\
& +\left(-W_{\mathrm{H}}-W_{\mathrm{O}}+3 A_{\mathrm{H}}+3 A_{\mathrm{O}}\right)\left\langle N_{\mathrm{H}} N_{\mathrm{O}}\right\rangle \\
& -\left(3 A_{\mathrm{H}}+A_{\mathrm{O}}\right)\left\langle N_{\mathrm{H}}^{2} N_{\mathrm{O}}\right\rangle \\
& -\left(A_{\mathrm{H}}+3 A_{\mathrm{O}}\right)\left\langle N_{\mathrm{H}} N_{\mathrm{O}}^{2}\right\rangle, \\
\mathrm{d}\left\langle N_{\mathrm{H}} N_{\mathrm{O}}\right\rangle &
\end{aligned}
$$

and the production rates of the molecular species are given by

$$
\begin{aligned}
& R_{\mathrm{H}_{2}}^{\text {grain }}=A_{\mathrm{H}}\left(\left\langle N_{\mathrm{H}}^{2}\right\rangle-\left\langle N_{\mathrm{H}}\right\rangle\right) \\
& R_{\mathrm{O}_{2}}^{\text {grain }}=A_{\mathrm{O}}\left(\left\langle N_{\mathrm{O}}^{2}\right\rangle-\left\langle N_{\mathrm{O}}\right\rangle\right) \\
& R_{\mathrm{OH}}^{\text {grain }}=\left(A_{\mathrm{H}}+A_{\mathrm{O}}\right)\left\langle N_{\mathrm{H}} N_{\mathrm{O}}\right\rangle .
\end{aligned}
$$

In the moment equations the time derivative of each moment is expressed as a linear combination of other moments. The set can be extended to include higher moments. However, the number of unknowns (namely moments to be computed) grows faster than the number of equations. In Eqs. (27), consisting of five equations, there are nine unknowns: $\left\langle N_{\mathrm{H}}\right\rangle,\left\langle N_{\mathrm{O}}\right\rangle,\left\langle N_{\mathrm{H}}^{2}\right\rangle$, $\left\langle N_{\mathrm{O}}^{2}\right\rangle,\left\langle N_{\mathrm{H}}^{3}\right\rangle,\left\langle N_{\mathrm{O}}^{3}\right\rangle,\left\langle N_{\mathrm{H}} N_{\mathrm{O}}\right\rangle,\left\langle N_{\mathrm{H}}^{2} N_{\mathrm{O}}\right\rangle$ and $\left\langle N_{\mathrm{H}} N_{\mathrm{O}}^{2}\right\rangle$.

Therefore, in order to solve the moment equations one has to use approximations for four of the unknowns listed above. In principle, these approximations can be obtained using the following properties. The number density of hydrogen in the gas phase is by orders of magnitude higher than that of any other specie, including oxygen. Therefore, $F_{\mathrm{H}} \gg F_{\mathrm{O}}$. The activation energies for diffusion and desorption of $\mathrm{H}$ atoms on the grain surface are much lower than those of heavier atomic species such as oxygen. Therefore, the diffusion of $\mathrm{H}$ atoms on the surface is much faster and dominates the chemical activity on the surface. The density of $\mathrm{H}$ atoms on the surface is thus primarily controlled by the processes of $\mathrm{H}_{2}$ formation and $\mathrm{H}$ desorption. The further reduction of $\mathrm{H}$ density due to the formation of $\mathrm{OH}$ can be considered as a small correction. We have examined such approximations and found that they do not work very well in practice and that the results deviate significantly from those of the master equation.

\section{Summary}

We have introduced a set of moment equations for the analysis of the formation of molecular hydrogen and other chemical reactions on dust grain surfaces in the interstellar medium. These equations are derived from the master equation that describes these processes. Like the master equation, the moment equations are exact even in the limit of small grains and low flux where the rate equations fail. They take into account the discrete nature of the populations of atomic and molecular species that participate in the reactions. While the master equation is expressed in terms of distributions such as $P_{\mathrm{H}}\left(N_{\mathrm{H}}\right)$, $N_{\mathrm{H}}=0,1,2 \ldots$, the moment equations are expressed in terms of the moments of these distributions.

We have shown that the moment equation approach is useful for simulations of molecular hydrogen formation. We expect that this approach will make it possible to efficiently calculate the total production rate of molecular hydrogen in diffuse clouds. The results will then be used in order to determine whether the molecular hydrogen formation process studied here is efficient enough to account for the observed abundance of $\mathrm{H}_{2}$. The input required for such calculations includes the surface parameters (obtained from laboratory experiments), the grain size distribution, the density and temperature of the $\mathrm{H}$ gas, the grain temperature as well as the dissociation rate of $\mathrm{H}_{2}$ molecules in the gas phase. Since the formation rate of 
molecular hydrogen is highly sensitive to the grain temperature and size distribution, the accuracy of the results may be limited by the quality of the observational data.

For more complex reaction networks involving multiple species, setting the cutoffs in the moment equations requires certain approximations that introduce significant errors. Therefore, for system that consist of more than a single reactive specie, we currently do not have a useful computational framework based on the moment equations. Potential advantages of the moment equations, justifying further attempts to further develop this approach, include the fact that they are similar in structure to the rate equations, possibly making it easier to incorporate them into models of interstellar chemistry. Moreover, they directly provide the first and second moments, which quantify the production rates of the molecular species involved. This is unlike the master equation in which the moments are calculated from the distribution in a post-processing stage.

Acknowledgements. We thank E. Herbst, V. Pirronello and G. Vidali for helpful discussions and correspondence. This work was supported by the Adler Foundation for Space Research of the Israel Science Foundation.

\section{References}

Aronowitz, S., \& Chang, S. B. 1985, ApJ, 293, 243

Bender, C. M., Brody, D. C., \& Meister, B. K. 2002, Inverse of a Vandermonde Matrix, preprint

Biham, O., Furman, I., Pirronello, V., \& Vidali, G. 2001, ApJ, 553, 595

Biham, O., \& Lipshtat, A. 2002, Phys. Rev. E, 66, 056103

Brackmann, R. T., \& Fite, W. L. 1961, J. Chem. Phys., 34, 1572

Brown, P. D. 1990, MNRAS, 243, 65

Brown, P. D., \& Charnley, S. B. 1990, MNRAS, 244, 432

Caselli, P., Hasegawa, T. I., \& Herbst, E. 1993, ApJ, 408, 548

Caselli, P., Hasegawa, T. I., \& Herbst, E. 1998, ApJ, 495, 309

Charnley, S. B. 2001, ApJ, 562, L99

Charnley, S. B., Tielens, A. G. G. M., \& Rodgers, S. D. 1997, ApJ, 482, L203

Cox, P., Boulanger, F., \& Jones, A. P. 2002, Dust in the Interstellar Medium, preprint

Draine, B. T., \& Lee, H. M. 1984, ApJ, 285, 89

Draine, B. T., \& Anderson, N. 1985, ApJ, 292, 494

Duley, W. W., \& Williams, D. A. 1986, MNRAS, 223, 177
Gould, R. J., \& Salpeter, E. E. 1963, ApJ, 138, 393

Green, N. J. B., Toniazzo, T., Pilling, M. J., et al. 2001, A\&A, 375, 1111

Hartquist, T. W., \& Williams, D. A. 1995, The chemically controlled cosmos (Cambridge: Cambridge University Press)

Hasegawa, T. I., Herbst, E., \& Leung, C. M. 1992, ApJS, 82, 167

Hasegawa, T. I., \& Herbst, E. 1993, MNRAS, 261, 83

Hasegawa, T. I., \& Herbst, E. 1993, MNRAS, 263, 589

d'Hendecourt, L. B., Allamandola, L. J., \& Greenberg, J. M. 1985, A\&A, 152, 130

Hollenbach, D., \& Salpeter, E. E. 1970, J. Chem. Phys., 53, 79

Hollenbach, D., \& Salpeter, E. E. 1971, ApJ, 163, 155

Hollenbach, E., Werner, M. W., \& Salpeter, E. E. 1971, ApJ, 163, 165

Katz, N., Furman, I., Biham, O., Pirronello, V., \& Vidali, G. 1999, ApJ, 522, 305

Landau, L. D., \& Lifshitz, E. M. 1980, Statistical Physics, Part I (Oxford: Pergamon Press)

Manicò, G., Ragunì, G., Pirronello, V., Roser, J. E., \& Vidali, G. 2001, ApJ, 548, L253

Mathis, J. S., Rumpl, W., \& Nordsieck, K. H. 1977, ApJ, 217, 425

Mathis, J. S. 1990, ARA\&A, 28, 37

Mathis, J. S. 1996, ApJ, 472, 643

Montroll, E. W., \& Weiss, G. H. 1965, J. Math. Phys., 6, 167

Pickles, J. B., \& Williams, D. A. 1977, Ap\&SS, 52, 433

Pirronello, V., \& Averna, D. 1988, A\&A, 201, 196

Pirronello, V., Liu, C., Shen, L., \& Vidali, G. 1997, ApJ, 475, L69

Pirronello, V., Biham, O., Liu, C., Shen, L., \& Vidali, G. 1997, ApJ, 483, L131

Pirronello, V., Liu, C., Roser, J. E., \& Vidali, G. 1999, A\&A, 344, 681

Sandford, S. A., \& Allamandola, L. J. 1993, ApJ, 409, L65

Schutte, A., Bassi, D., Tommasini, F., et al. 1976, J. Chem Phys., 64, 4135

Shalabiea, O. M., \& Greenberg, J. M. 1994, A\&A, 290, 266

Shalabiea, O. M., Caselli, P., \& Herbst, E. 1998, ApJ, 502, 652

Smoluchowski, R. 1981, Ap\&SS, 75, 353

Smoluchowski, R. 1983, J. Chem. Phys., 87, 4229

Stantcheva, T., Caselli, P., \& Herbst, E. 2001, A\&A, 375, 673

Stantcheva, T., Shematovich, V. I., \& Herbst, E. 2002, A\&A, 391, 1069

Stantcheva, T., \& Herbst, E. 2002, Deuterium fractionation on interstellar grains studied with the direct master equation approach, preprint

Takahashi, J., Masuda, K., \& Nagaoka, M. 1999, MNRAS, 306, 22

Tielens, A. G. G. M. 1995, unpublished

Weingartner, J. C., \& Draine, B. T. 2001, ApJ, 548, 296

Willacy, K., \& Williams, D. A. 1993, MNRAS, 260, 635

Williams, D. A. 1968, ApJ, 151, 935 